\title{
Radiation therapy for pelvic recurrent colorectal or gynecological cancer: is whole pelvic irradiation necessary?
}

\author{
Naoya Ishibashi ${ }^{1}$, Toshiya Maebayashi ${ }^{1}$, Masaharu Hata ${ }^{2}$, Takuya Aizawa ${ }^{1}$, Masakuni Sakaguchi ${ }^{1}$, \\ Masahiro Okada ${ }^{1}$ \\ ${ }^{1}$ Department of Radiology, Nihon University School of Medicine, Itabashi-ku, Tokyo, Japan; ${ }^{2}$ Department of Radiation Oncology, Yokohama City \\ University Graduate School of Medicine, Yokohama-shi, Kanagawa, Japan \\ Contributions: (I) Conception and Design: N Ishibashi; (II) Administrative support: None; (III) Provision of study materials or patients: All authors; \\ (IV) Collection and assembly of data: All authors; (V) Data analysis and interpretation: N Ishibashi; (VI) Manuscript writing: All authors; (VII) Final \\ approval of the manuscript: All authors. \\ Correspondence to: Naoya Ishibashi. Department of Radiology, Nihon University School of Medicine, 30-1 Oyaguchi Kami-cho, Itabashi-ku, Tokyo \\ 173-8610, Japan. Email: ishibashi.naoya@nihon-u.ac.jp.
}

Background: Preoperative whole pelvic radiation therapy (RT) is used commonly for rectal cancer and is the standard field postoperatively in gynecological cancer. However, the ideal field (local vs. whole pelvis) has not been determined for local recurrence of these cancers.

Methods: We retrospectively reviewed the data for 52 patients who developed local tumor recurrence of rectal or gynecological cancer treated from 2013 to 2021. The initial treatment for all patients was total excision of the primary tumors without radiation therapy. Radiation therapy targets were surgical stumps, perianastomosis sites, and pelvic lymph nodes, classified according to the pelvic nodal volume atlas for radiation therapy. Patients were divided into the local recurrent tumor only radiation therapy group and the whole pelvis radiation therapy group. Whole pelvis radiation therapy included the common iliac lymph nodes or prophylactic lymph nodes below the L5/S1 junction. We recorded second recurrence after RT and the affected site(s) in each group. We also compared disease-specific survival using uni- and multivariate analyses.

Results: We found no significant differences between the groups regarding second recurrence or regarding the site(s) of recurrence. We also found no significant differences in disease-specific survival between the two RT groups. However, patients who did not receive chemotherapy after the initial surgery and before RT had significantly longer survival $(\mathrm{P}=0.015)$.

Conclusions: In patients with locally recurrent rectal or gynecological cancer, we found no significant difference in second recurrence or survival between the local tumor only RT field and the whole pelvic RT field.

Keywords: Radiation therapy (RT); pelvic recurrent tumor; colorectal cancer; gynecological cancer; whole pelvic radiation field

Submitted Oct 13, 2021. Accepted for publication Jan 27, 2022.

doi: 10.21037/apm-21-2950

View this article at: https://dx.doi.org/10.21037/apm-21-2950

\section{Introduction}

Since total mesorectal excision (TME) has become the standard of care for treating rectal cancer, the postoperative local recurrence (LR) rate of rectal cancer has decreased to $11.5-12.6 \%(1,2)$. The postoperative LR rate of uterine endometrial cancer ranges from $14 \%$ to $18 \%(3,4)$, while the LR rate for uterine cervical cancer after surgery or radiation therapy (RT) ranges from $7.2 \%$ to $12.5 \%(5,6)$. The National Comprehensive Cancer Network guidelines recommend RT as a treatment for LR (7-9). A whole pelvic radiation field, which includes the prophylactic lymph node (LN) regions, is the major field for preoperative RT for 
rectal cancer and the standard field for postoperative RT for endometrial and cervical cancers (10-13). However, no RT fields have been identified for RT for LR. The significance of covering the whole pelvic radiation field, including the prophylactic LN regions, is unclear. Additionally, the frequency of second LR in out-field portions of the pelvis after RT is delivered to an RT field that includes the local recurrent tumor only, without the prophylactic $\mathrm{LN}$ regions, is unknown. Recently, pelvic RT fields for colorectal or gynecological cancer have been determined using definitions presented in the pelvic nodal volume atlas (14-16). In the present study, local recurrent tumor locations were meticulously classified according to the definitions presented in the atlas, and sites of second LR after RT were compared between RT with an RT field including only the local recurrent tumor, and RT with a whole pelvic radiation field. To our knowledge, this is the first study comparing these RT fields in patients with second LR after RT. We present the following article in accordance with the STROBE reporting checklist (available at https://apm. amegroups.com/article/view/10.21037/apm-21-2950/rc).

\section{Methods}

We retrospectively reviewed the data for 52 patients with pelvic local recurrent tumor of colorectal or gynecological cancer treated with RT between 2013 and 2021. The study was conducted in accordance with the Declaration of Helsinki (as revised in 2013). This retrospective study was approved by the Institutional Review Board of Nihon University School of Medicine (No. RK-211109-6). Written informed consent for publication was obtained from the patients before RT. As initial treatment, all patients underwent total excision of the primary tumors, only, and did not receive RT. Only one patient with sigmoid colon cancer was suspected to have residual tumors at the perianastomosis site during the initial surgery and included this study. Regarding local recurrent tumor locations, the RT targets of stumps, perianastomosis sites, and pelvic LNs were meticulously classified according to definitions presented in the pelvic nodal volume atlas for RT (14-16). The location of local recurrent tumors in mesorectal LNs after TME of rectal cancer was defined as the area where the mesorectum was located before surgery. When local recurrent tumors invaded the surrounding organs, the surrounding organs were also included in the RT targets. We divided the RT fields into two groups: the local recurrent tumor only group and the whole pelvis group, which included the common iliac LNs or prophylactic $\mathrm{LN}$ regions below the L5/S1 junction. The gross tumor volume (GTV) was defined as the local recurrent tumor, and the planned target volume constituted at least a $5-\mathrm{mm}$ margin around the GTV. All patients were irradiated using three-dimensional conformal RT. In patients in the local recurrent tumor only group, the presence of concurrent other pelvic LN metastases outside the RT field was defined as concurrent other site metastasis at RT. Local tumor response after RT was evaluated according to the Response Evaluation Criteria in Solid Tumors (17).

\section{Statistical methods}

SPSS version 21.0 (IBM Corp., Armonk, NY, USA) was used for statistical analysis. Univariate analysis using Pearson's $\chi^{2}$ test was performed to analyze the presence or absence of a second recurrence after RT and the sites of the second recurrence in the two RT field groups. The Kaplan-Meier method was used to calculate disease-specific survival (DSS) from the date of RT completion. Differences in DSS between subgroups were analyzed using the univariate log-rank test and a multivariate Cox proportional hazard model for the following patient characteristics: age (< median $v s . \geq$ median), primary tumor site (colorectal $v s$. gynecological), days from initial surgery to RT ( $<$ median $v s . \geq$ median), chemotherapy between the initial surgery and RT (yes vs. no), concurrent other site metastasis at RT (yes vs. no), Eastern Cooperative Oncology Group (ECOG) performance status at RT (0-1 vs. $\geq 2)$, RT field group (local recurrent tumor only $v s$. whole pelvis), and chemotherapy after RT (yes vs. no). Differences with P values $<0.05$ were considered statistically significant.

\section{Results}

\section{Patient characteristics}

The clinical data of all 52 patients are summarized in Table 1; 17 (32.7\%) were male, and 35 (67.3\%) were female. The age at RT initiation ranged from 32 to 88 years (median, 67 years). The most common site of local recurrent tumors targeted by RT was the region including stumps in 22 patients $(42.3 \%)$, followed by the region including mesorectal LNs in 7 patients (13.5\%). The most common primary tumor was rectal cancer, which was found in 18 patients (34.6\%), followed by colon and uterine endometrial cancers in 10 patients $(19.2 \%)$. One 
Table 1 Characteristics of patients undergoing radiation therapy for pelvic local recurrent tumors $(\mathrm{n}=52)$

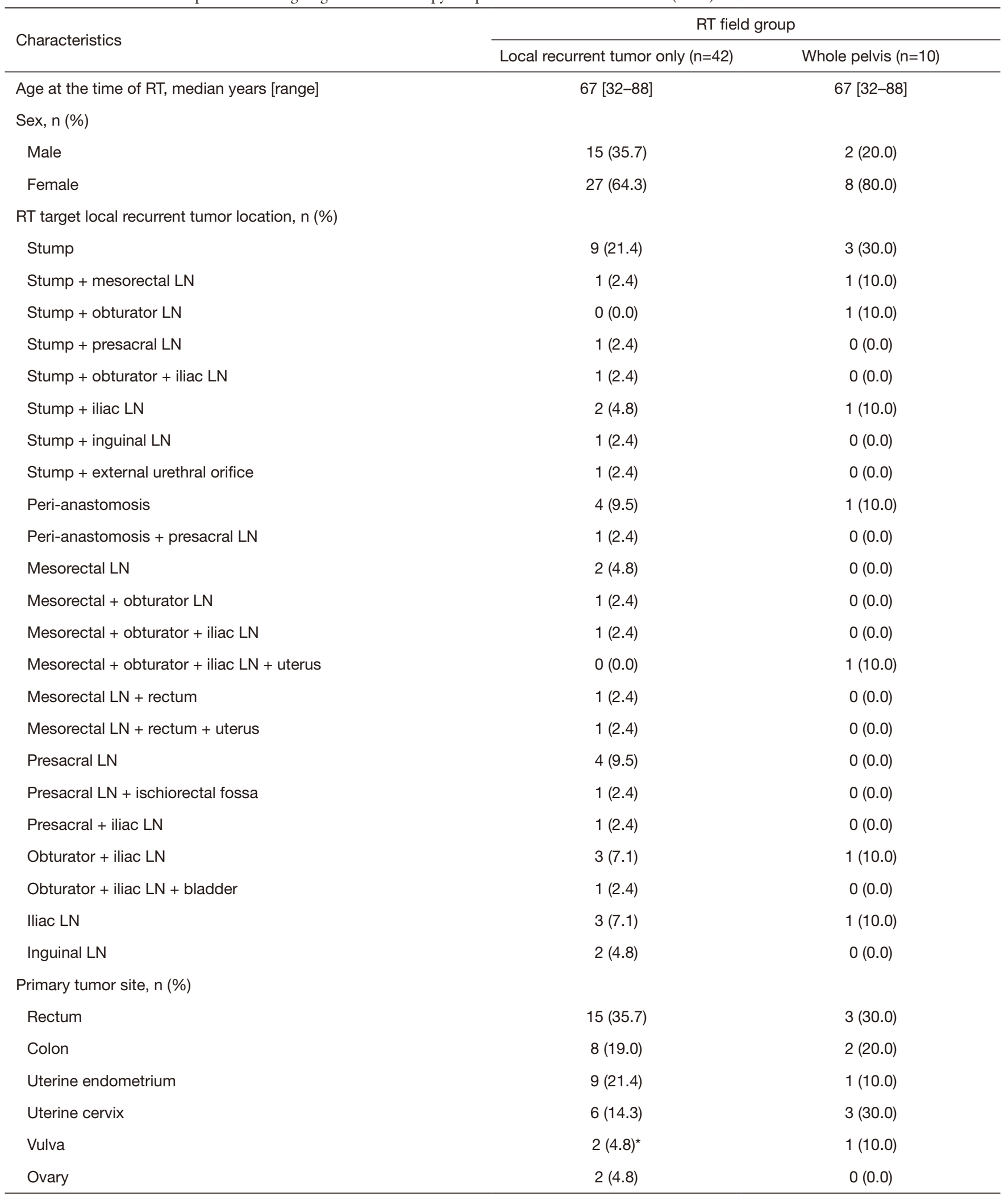

Table 1 (continued) 
Table 1 (continued)

\begin{tabular}{|c|c|c|}
\hline Characteristics & \multicolumn{2}{|c|}{ RT field group } \\
\hline The days from initial surgery to RT, median days [range] & $559[51-3,748]$ & $704[149-2,231]$ \\
\hline \multicolumn{3}{|l|}{ Chemotherapy between initial surgery and RT, n (\%) } \\
\hline Yes & $29(69.0)$ & $6(60.0)$ \\
\hline \multicolumn{3}{|l|}{ Concurrent other site metastasis at RT, n (\%) } \\
\hline Yes & $19(45.2)$ & $1(10.0)$ \\
\hline No & $23(54.8)$ & $9(90.0)$ \\
\hline \multicolumn{3}{|l|}{ ECOG performance status at RT, n (\%) } \\
\hline 3 & $2(4.8)$ & $0(0.0)$ \\
\hline \multicolumn{3}{|l|}{ Purpose of RT, n (\%) } \\
\hline Pain relief & $16(38.1)$ & $4(40.0)$ \\
\hline Hemostasis & $4(9.5)$ & $0(0.0)$ \\
\hline Leg edema release & $2(4.8)$ & $1(10.0)$ \\
\hline No symptom & $20(47.6)$ & $5(50.0)$ \\
\hline \multicolumn{3}{|l|}{ RT field group and total median dose in Gy (range), n (\%) } \\
\hline Local tumor only, 50.4 (30.0-66.0) & $40(95.2)$ & - \\
\hline Yes & $16(38.1)$ & $4(40.0)$ \\
\hline No & $26(61.9)$ & $6(60.0)$ \\
\hline \multicolumn{3}{|l|}{ Chemotherapy after RT, n (\%) } \\
\hline Yes & $22(52.4)$ & $7(70.0)$ \\
\hline No & $20(47.6)$ & $3(30.0)$ \\
\hline
\end{tabular}

*, including one extramammary Paget's disease. RT, radiation therapy; LN, lymph node; ECOG, Eastern Cooperative Oncology Group; EBRT, external beam radiation therapy; BT, brachytherapy.

patient with inguinal LN metastases from ovarian cancer treated with RT was reported before (18). The days from initial surgery to RT ranged from 51 to 3,748 days (median, 567 days). Chemotherapy was administered between the initial surgery and RT in 35 patients $(67.3 \%)$ and 29 patients $(55.8 \%)$ received adjuvant chemotherapy after initial surgery because of high risk of recurrence such as LN metastasis. Nine patients received bevacizumab between the initial surgery and RT and five patients received bevacizumab after RT. Concurrent other site metastasis at 
Table 2 Treatment outcome after radiation therapy for pelvic recurrent tumors

\begin{tabular}{lc}
\hline Parameter & Number (\%) \\
\hline Tumor response & $15(28.8)$ \\
Complete response & $3(5.8)$ \\
Partial response & $18(34.6)$ \\
Stable disease & $16(30.8)$ \\
Progressive disease & $5(9.6)$ \\
Abscess formation & \\
Symptom relief (n=27) & $2(7.4)$ \\
Complete relief** & $19(70.4)$ \\
Partial relief & $6(22.2)$ \\
No relief & \\
\hline
\end{tabular}

${ }^{*}$, five patients developed progressive disease with abscess formation; ${ }^{* *}$, both patients achieved hemostasis.

RT occurred in 20 patients (38.5\%). The most common purpose of RT was local control of asymptomatic LR in 25 patients (48.1\%), followed by pain relief in 20 patients (38.5\%). Regarding the RT field groups, 42 patients (80.8\%) received irradiation to the local recurrent tumors only, and 10 patients (19.2\%) received irradiation to the whole pelvis. This study was retrospective, so no rationale of selection radiation field was ruled. Regarding the RT total dose, external beam RT (EBRT) at a median dose of 50.4 Gy was delivered to 40 patients in the local recurrent tumor only group, while EBRT at a median dose of 45.0 Gy with a vaginal brachytherapy (BT) boost of 10 Gy was delivered to the stumps of endometrial carcinoma in 2 patients. In the whole pelvis group, EBRT at a dose of 45.0 Gy was delivered to one patient, EBRT at a median dose of 45.0 Gy with a median EBRT boost of 10.0 Gy was delivered to seven patients, and EBRT at a median dose of 50.2 Gy with a median vaginal BT boost of $17.0 \mathrm{~Gy}$ was delivered to the stumps of cervical carcinoma or the stumps plus obturator LNs in two patients, respectively. Concurrent chemotherapy during RT was administered to 10 patients (19.2\%).

\section{Local response and symptom relief}

The tumor local response after RT was evaluated by computed tomography (CT) at a median of 402 days (range, 1-2,572 days) from the date of RT completion. An overall response was observed in $18(34.6 \%)$ patients, and a complete response was observed in 15 (28.8\%) patients. Progressive disease was observed in 16 patients (30.8\%), among whom 5 (9.6\%) showed abscess formation on CT (Table 2). Abscess formation was observed in all patients with locally recurrent colorectal cancer (three patients with colon cancer and two patients with rectal cancer). In three of the five patients received irradiation to the local recurrent tumors only, and the other two patients received irradiation to the whole pelvis. RT was delivered at a total dose of 30-61.2 Gy, while tumors were irradiated with 1.8-3 Gy per fraction. In one of the five patients, the rectum was included in the RT targets because rectal invasion was suspected before RT. Three of the five patients with abscess formation received anti-vascular endothelial growth factor agent. Two patients received bevacizumab before or after RT and one received aflibercept with and after RT. Two patients with stable disease underwent total excision of local recurrent tumors after RT. Among 27 symptomatic patients at the start of RT, 2 (7.4\%) experienced complete relief, and hemostasis was achieved in all patients. Partial symptom relief was achieved in 19 (70.4\%) patients (Table 2).

\section{Acute toxicity}

Regarding grade $\geq 3$ RT-related toxicities (National Cancer Institute Common Terminology Criteria for Adverse Events 5.0) (19), hematological toxicity was observed in only one patient.

\section{Second recurrent site after $R T$, and survival}

The second recurrence after RT delivered to the first local recurrent tumors was evaluated using CT, in principle which was performed to evaluate the local responses after RT. A second recurrence after RT delivered to the first local recurrent tumor was observed in 27 patients (51.9\%). The most common site of second recurrence was extrapelvic metastasis in 13 patients $(25.0 \%)$. Among 42 patients in the local recurrent tumor only group, $12(28.6 \%)$ had new pelvic LN recurrence. Among 10 patients in the whole pelvis group, 2 (20.0\%) had new pelvic LN recurrence. In the local recurrent tumor only group, 9 patients $(21.4 \%)$ had new extra-pelvic metastatic recurrence only. In the whole pelvis group, 4 patients $(40.0 \%)$ had new extra-pelvic metastatic recurrence only. No significant differences in the presence or absence of a second recurrence as well as the sites were observed between the two RT field groups (Table 3). In the local recurrent tumor only group, the cumulative 
Table 3 Second recurrent site after radiation therapy for pelvic recurrent tumors and univariate analyses of the associations between recurrence and the RT field

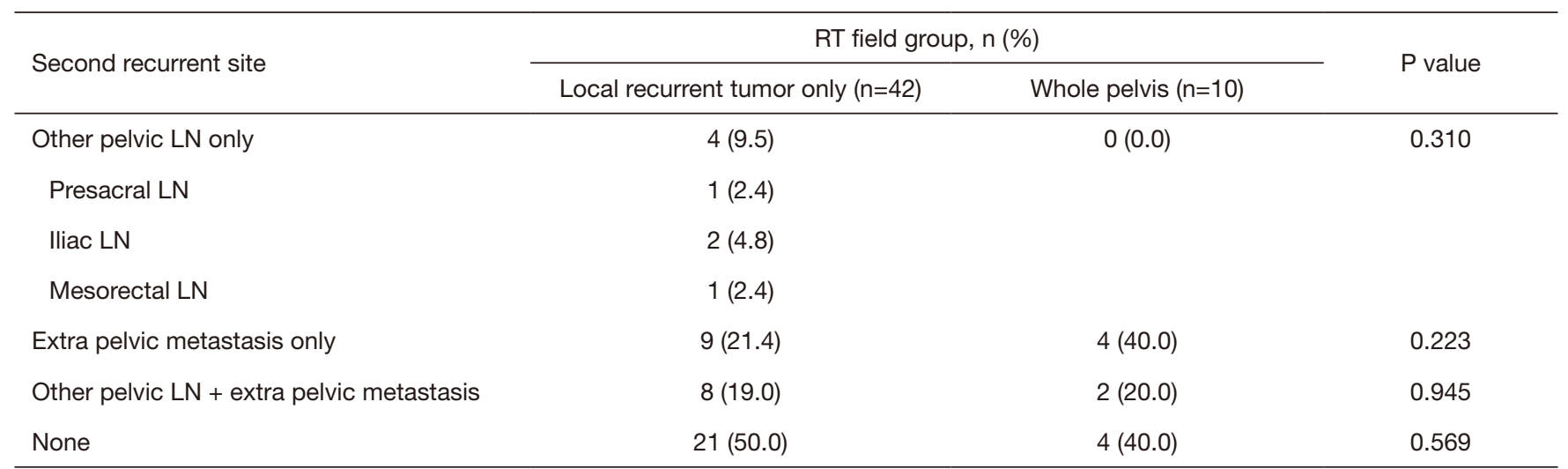

$\mathrm{RT}$, radiation therapy; LN, lymph node.

Table 4 Univariate and multivariate analyses of the predictors of disease-specific survival after radiation therapy for pelvic recurrent tumors

\begin{tabular}{|c|c|c|c|c|}
\hline Prognostic factor & Characteristics & $\frac{\text { Univariate analysis }}{\mathrm{P} \text { value }}$ & \multicolumn{2}{|c|}{ Multivariate analysis } \\
\hline Age at RT & $<67$ vs. $\geq 67$ years & 0.105 & $0.441(0.195-0.988)$ & $0.049^{*}$ \\
\hline Primary tumor site & Colorectal vs. gynecological & 0.083 & $4.253(1.143-15.831)$ & $0.031^{*}$ \\
\hline Days from initial surgery to RT & $<567$ vs. $\geq 567$ days & 0.567 & $0.356(0.075-1.682)$ & 0.192 \\
\hline Concurrent other site metastasis at RT & Yes vs. no & $0.000431^{*}$ & 6.059 (2.163-16.968) & $0.001^{*}$ \\
\hline ECOG performance status at RT & $0-1$ vs. $2-3$ & 0.093 & $1.986(0.700-5.640)$ & 0.197 \\
\hline RT field group & $\begin{array}{l}\text { Local recurrent tumor only vs. } \\
\text { whole pelvis }\end{array}$ & 0.745 & $2.695(0.892-8.145)$ & 0.079 \\
\hline
\end{tabular}

*, significant difference between the groups $(\mathrm{P}<0.05)$. $\mathrm{Cl}$, confidence interval; $\mathrm{RT}$, radiation therapy; ECOG, Eastern Cooperative Oncology Group.

DSS rates were $62.1 \%$ [standard error $(\mathrm{SE}) \pm 7.8 \%$ ] 1 year after $\mathrm{RT}$ and $43.9 \%(\mathrm{SE} \pm 8.3 \%) 3$ years after $\mathrm{RT}$. In the whole pelvis group, the cumulative DSS rates were $80.0 \%$ $(\mathrm{SE} \pm 12.6 \%) 1$ year after $\mathrm{RT}$ and $40.0 \%(\mathrm{SE} \pm 15.5 \%)$ 3 years after RT. Table 4 lists the results of univariate and multivariate analyses for associations between the patients' characteristics and prognosis. No significant differences in DSS were observed between the two RT field groups (Table 4). Multivariate analyses identified gynecological cancer, no chemotherapy between initial surgery and $\mathrm{RT}$, and no concurrent metastasis to other sites at RT as significant factors for favorable DSS (Table 4). Figures 1-3 summarize the comparisons of DSS between the local recurrent tumor only group and whole pelvis group; chemotherapy between initial surgery and RT (yes vs. no); and concurrent other site metastasis at RT (yes vs. no).

\section{Discussion}

Postoperative LR of rectal cancer has been treated with RT for many years. In the past, the RT field was the whole pelvis, which included prophylactic $\mathrm{LN}$ regions centered on two parallel opposite anteroposterior and posteroanterior standard pelvic fields $(20,21)$. In-field second LR after RT was observed in $56.6 \%$ of patients, in one study (20). The RT field for postoperative LR of uterine endometrial cancer 


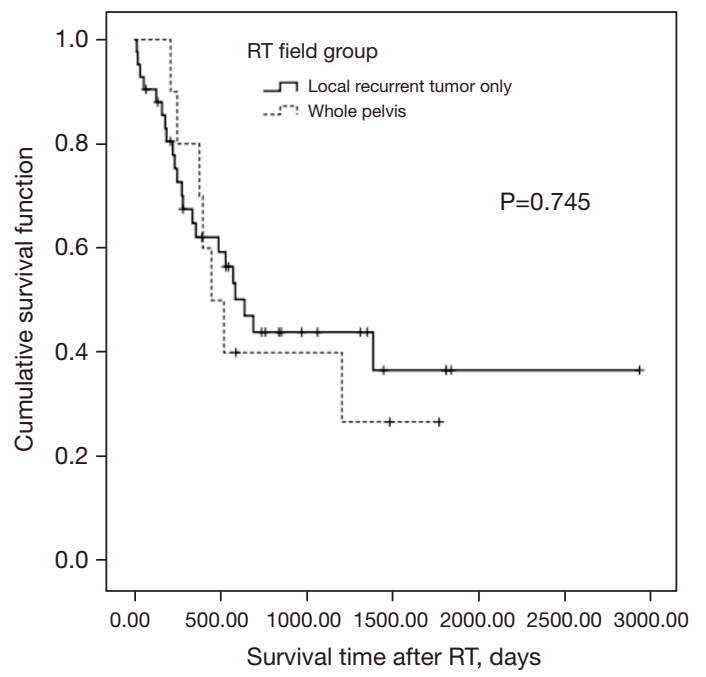

Figure 1 Kaplan-Meier survival curves for patients undergoing radiation therapy for the local recurrent tumor only $v s$. the whole pelvis. The difference between the groups was not statistically significant $(\mathrm{P}=0.745)$.

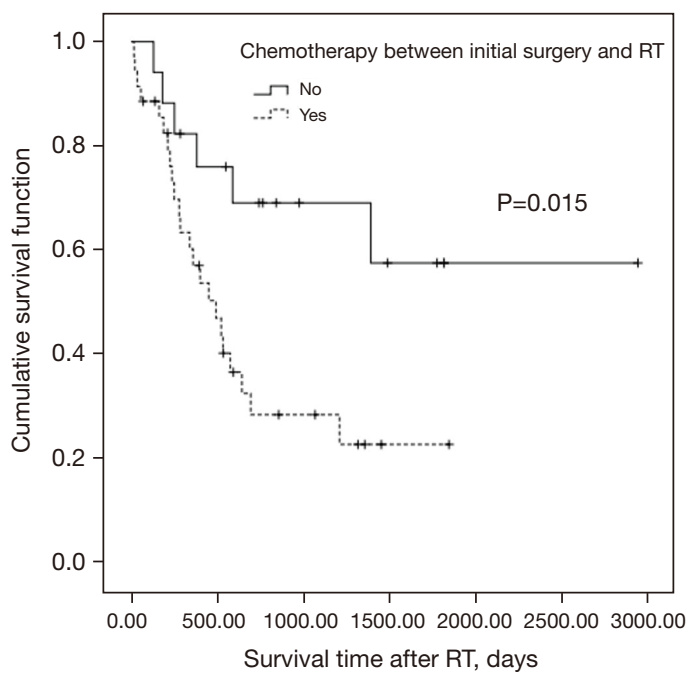

Figure 2 Kaplan-Meier survival curves for patients treated with or without chemotherapy between the initial surgery and radiation therapy (yes vs. no). Survival was significantly longer in patients who did not receive chemotherapy $(\mathrm{P}=0.015)$. $\mathrm{RT}$, radiation therapy.

is also the whole pelvis, including the prophylactic $\mathrm{LN}$ regions, and in some patients, a vaginal BT boost is often combined with RT (22-24). Studies have reported that infield second LR after RT was observed in $7.1-15.1 \%$ of patients $(23,24)$. In a study that included patients receiving

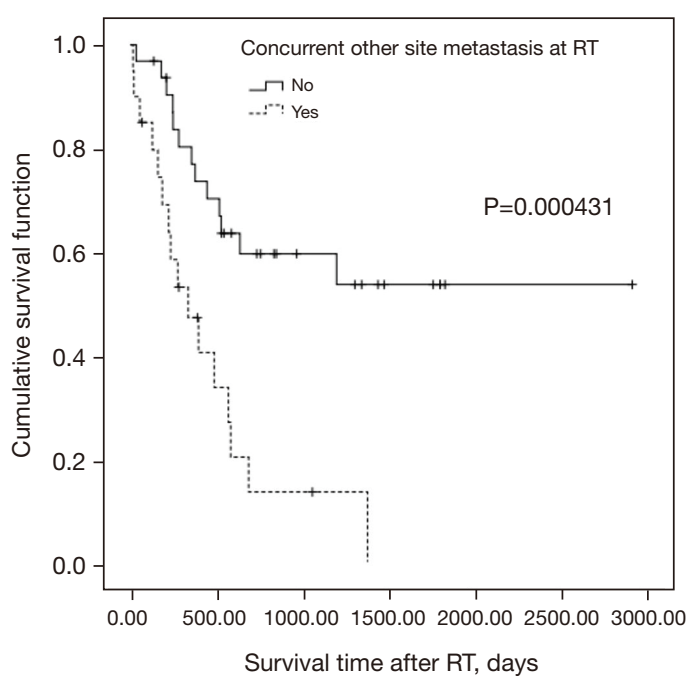

Figure 3 Kaplan-Meier survival curves for patients with or without concurrent other site metastasis at radiation therapy. Survival was significantly longer in patients without concurrent other site metastasis at RT $(\mathrm{P}=0.000431)$. RT, radiation therapy.

vaginal BT with and without whole pelvic RT, pelvic progressive disease was observed in $52.1 \%$ of the patients after RT (22). In contrast, few studies have evaluated second LR after RT in patients for which RT was delivered to fields that included local recurrent tumors only instead of the whole pelvic radiation field. In the present study, most patients received irradiation to an RT field that included local recurrent tumors only, and $28.5 \%$ of the patients in the local recurrent tumor only group had other pelvic LN recurrences. In addition, we confirmed for the first time that no significant differences were observed regarding the presence or absence of second recurrence, second recurrent sites, or DSS between the local recurrent tumor only group and the whole pelvis group. One limitation of this study was that the number of patients treated with whole pelvis was small. The extension of RT field to the pelvic nodes may be detrimental for the patients treated with palliative intent. Because of recent advances in RT, intensity-modified RT (IMRT) delivered to the whole pelvis to reduce the toxicity has been reported, including the preoperative rectal cancer or local recurrent rectal cancer and prophylactic $\mathrm{LN}$ regions $(25,26)$. When IMRT was administered with concurrent chemotherapy to the local recurrent rectal cancer, the local control rate (combined complete and partial responses) was $46.5 \%$ (26). In the present study, the local control rate (combined complete and partial responses) was $34.6 \%$, which was lower than the rate mentioned 
above. This appears to be attributable to the following reasons: the present study included many patients with LR with concurrent metastasis to other sites at RT. In many patients, RT was administered at palliative doses to achieve symptom relief instead of at definitive RT doses, and most patients received RT without concurrent chemotherapy. Local recurrent tumors of colorectal or gynecological cancer are refractory to RT, which is explained by the more hypoxic nature of local recurrent tumors compared with primary tumors (27). In the present study, DSS was longer in patients who did not receive chemotherapy between the initial surgery and RT than in those who received RT after chemotherapy. This suggests that chemotherapy might have caused local recurrent tumors to become more hypoxic.

Recent reports have described stereotactic body RT (SBRT) or carbon ion RT administered at an increased RT dose per fraction to irradiate local tumors only at the highest RT dose possible, in patients without concurrent metastasis to other sites at RT. These therapies result in favorable treatment outcomes as indicated by the local control rate of $64.5-88.0 \%$ (28-30). In comparison, the present study reported a 3 -year DSS rate of $40.0-43.9 \%$, which was better than the rate of $27-36.5 \%$ in previous reports of IMRT and SBRT $(26,28)$. This suggests that conventional EBRT may be sufficient to affect the survival rate in LR. In SBRT at a high RT dose per fraction, toxicities, such as colorectal perforation and rectovaginal fistula have been reported in $13.0-14.3 \%$ of patients $(28,29)$. In the present study, as local recurrent tumors proliferated after RT, abscess formation was observed in $9.6 \%$ of the patients. Because the total RT dose delivered to these patients was not as high as that with SBRT, abscess formation was presumably caused by colorectal perforation owing to invasion of proliferating local recurrent tumors rather than because of toxicity. All patients with abscesses had locally recurrent colorectal cancer. Thus, caution should be exercised regarding colorectal perforation if local recurrent tumors proliferate after RT in patients who undergo intestinal tract resection at the initial surgery. In the future, we will examine abscess formation after RT in patients with local recurrent tumors who receive RT and we will also investigate the optimal dose of conventional EBRT for local recurrent tumors in patients with concurrent metastasis at other sites at RT.

\section{Acknowledgments}

We thank Jane Charbonneau, DVM, from Edanz (https:// jp.edanz.com/ac) for editing a draft of this manuscript. Funding: None.

\section{Footnote}

Reporting Checklist: The authors have completed the STROBE reporting checklist. Available at https://apm. amegroups.com/article/view/10.21037/apm-21-2950/rc

Data Sharing Statement: Available at https://apm.amegroups. com/article/view/10.21037/apm-21-2950/dss

Conflicts of Interest: All authors have completed the ICMJE uniform disclosure form (available at https://apm. amegroups.com/article/view/10.21037/apm-21-2950/coif). The authors have no conflicts of interest to declare.

Ethical Statement: The authors are accountable for all aspects of the work in ensuring that questions related to the accuracy or integrity of any part of the work are appropriately investigated and resolved. The study was conducted in accordance with the Declaration of Helsinki (as revised in 2013). This retrospective study was approved by the Institutional Review Board of Nihon University School of Medicine (No. RK-211109-6). Written informed consent for publication was obtained from the patients before radiation therapy.

Open Access Statement: This is an Open Access article distributed in accordance with the Creative Commons Attribution-NonCommercial-NoDerivs 4.0 International License (CC BY-NC-ND 4.0), which permits the noncommercial replication and distribution of the article with the strict proviso that no changes or edits are made and the original work is properly cited (including links to both the formal publication through the relevant DOI and the license). See: https://creativecommons.org/licenses/by-nc-nd/4.0/.

\section{References}

1. Bolognese A, Cardi M, Muttillo IA, et al. Total mesorectal excision for surgical treatment of rectal cancer. J Surg Oncol 2000;74:21-3.

2. van den Brink $M$, Stiggelbout $A M$, van den Hout $W B$, et al. Clinical nature and prognosis of locally recurrent rectal cancer after total mesorectal excision with or without preoperative radiotherapy. J Clin Oncol 2004;22:3958-64.

3. Creutzberg CL, van Putten WL, Koper PC, et al. Surgery 
and postoperative radiotherapy versus surgery alone for patients with stage-1 endometrial carcinoma: multicentre randomised trial. PORTEC Study Group. Post Operative Radiation Therapy in Endometrial Carcinoma. Lancet 2000;355:1404-11.

4. Randall ME, Filiaci VL, Muss H, et al. Randomized phase III trial of whole-abdominal irradiation versus doxorubicin and cisplatin chemotherapy in advanced endometrial carcinoma: a Gynecologic Oncology Group Study. J Clin Oncol 2006;24:36-44.

5. Hong JH, Tsai CS, Lai CH, et al. Recurrent squamous cell carcinoma of cervix after definitive radiotherapy. Int J Radiat Oncol Biol Phys 2004;60:249-57.

6. Yoshida K, Kajiyama H, Utsumi F, et al. A post-recurrence survival-predicting indicator for cervical cancer from the analysis of 165 patients who developed recurrence. Mol Clin Oncol 2018;8:281-5.

7. NCCN Clinical Practice Guidelines in Oncology (NCCN Guidelines®). Rectal Cancer. Version 1. 2021.

8. NCCN Clinical Practice Guidelines in Oncology (NCCN Guidelines®). Endometrial Carcinoma. Version 3. 2021.

9. NCCN Clinical Practice Guidelines in Oncology (NCCN Guidelines®). Cervical Cancer. Version 1. 2021.

10. Kapiteijn E, Kranenbarg EK, Steup WH, et al. Total mesorectal excision (TME) with or without preoperative radiotherapy in the treatment of primary rectal cancer. Prospective randomised trial with standard operative and histopathological techniques. Dutch ColoRectal Cancer Group. Eur J Surg 1999;165:410-20.

11. Gérard JP, Conroy T, Bonnetain F, et al. Preoperative radiotherapy with or without concurrent fluorouracil and leucovorin in T3-4 rectal cancers: results of FFCD 9203. J Clin Oncol 2006;24:4620-5.

12. Creutzberg CL, Nout RA, Lybeert ML, et al. Fifteen-year radiotherapy outcomes of the randomized PORTEC-1 trial for endometrial carcinoma. Int J Radiat Oncol Biol Phys 2011;81:e631-8.

13. Peters WA 3rd, Liu PY, Barrett RJ 2nd, et al. Concurrent chemotherapy and pelvic radiation therapy compared with pelvic radiation therapy alone as adjuvant therapy after radical surgery in high-risk early-stage cancer of the cervix. J Clin Oncol 2000;18:1606-13.

14. Roels S, Duthoy W, Haustermans K, et al. Definition and delineation of the clinical target volume for rectal cancer. Int J Radiat Oncol Biol Phys 2006;65:1129-42.

15. Ng M, Leong T, Chander S, et al. Australasian Gastrointestinal Trials Group (AGITG) contouring atlas and planning guidelines for intensity-modulated radiotherapy in anal cancer. Int J Radiat Oncol Biol Phys 2012;83:1455-62.

16. Japan Clinical Oncology Group; Toita T, Ohno T, et al. A consensus-based guideline defining the clinical target volume for pelvic lymph nodes in external beam radiotherapy for uterine cervical cancer. Jpn J Clin Oncol 2010;40:456-63.

17. Eisenhauer EA, Therasse P, Bogaerts J, et al. New response evaluation criteria in solid tumours: revised RECIST guideline (version 1.1). Eur J Cancer 2009;45:228-47.

18. Ishibashi N, Maebayashi T, Nishimaki H, et al. Radiation therapy for superficial inguinal lymph node metastases from ovarian clear cell carcinoma and associated inguinal hernia. Transl Cancer Res 2018;7:1326-30.

19. National Cancer Institute Common Terminology Criteria for Adverse Events (CTCAE) 5.0. Nov 27, 2017.

20. Lybeert ML, Martijn H, de Neve W, et al. Radiotherapy for locoregional relapses of rectal carcinoma after initial radical surgery: definite but limited influence on relapsefree survival and survival. Int J Radiat Oncol Biol Phys 1992;24:241-6.

21. Knol HP, Hanssens PE, Rutten HJ, et al. Effect of radiation therapy alone or in combination with surgery and/or chemotherapy on tumor and symptom control of recurrent rectal cancer. Strahlenther Onkol 1997;173:43-9.

22. Jereczek-Fossa B, Badzio A, Jassem J. Recurrent endometrial cancer after surgery alone: results of salvage radiotherapy. Int J Radiat Oncol Biol Phys 2000;48:405-13.

23. Arden JD, Gruner MF, Vu CC, et al. Outcomes After Salvage Radiation Therapy for Recurrent Endometrial Cancer in Patients With No Prior Adjuvant Therapy: An Institutional Review. Adv Radiat Oncol 2020;5:1240-7.

24. Lindemann K, Smogeli E, Småstuen MC, et al. Salvage Radiation for Pelvic Relapse after Surgically Treated Endometrial Cancer. Cancers (Basel) 2021;13:1367.

25. Geng JH, Zhang YZ, Li YH, et al. Preliminary results of simultaneous integrated boost intensity-modulated radiation therapy based neoadjuvant chemoradiotherapy on locally advanced rectal cancer with clinically suspected positive lateral pelvic lymph nodes. Ann Transl Med 2021;9:217.

26. Cai G, Zhu J, Palmer JD, et al. CAPIRI-IMRT: a phase II study of concurrent capecitabine and irinotecan with intensity-modulated radiation therapy for the treatment of recurrent rectal cancer. Radiat Oncol 2015;10:57.

27. Höckel M, Schlenger K, Höckel S, et al. Tumor hypoxia in pelvic recurrences of cervical cancer. Int J Cancer 1998;79:365-9. 
28. Seo Y, Kim MS, Yoo HJ, et al. Salvage stereotactic body radiotherapy for locally recurrent uterine cervix cancer at the pelvic sidewall: Feasibility and complication. Asia Pac J Clin Oncol 2016;12:e280-8.

29. Cheng HY, Liang JA, Hung YC, et al. Stereotactic body radiotherapy for pelvic boost in gynecological cancer patients with local recurrence or unsuitable for intracavitary brachytherapy. Taiwan J Obstet Gynecol 2021;60:111-8.

30. Yamada S, Kamada T, Ebner DK, et al. Carbon-Ion Radiation Therapy for Pelvic Recurrence of Rectal Cancer. Int J Radiat Oncol Biol Phys 2016;96:93-101.

Cite this article as: Ishibashi N, Maebayashi T, Hata $M$, Aizawa T, Sakaguchi M, Okada M. Radiation therapy for pelvic recurrent colorectal or gynecological cancer: is whole pelvic irradiation necessary? Ann Palliat Med 2022;11(6):1855-1864. doi: 10.21037/apm-21-2950 